\title{
Numerical study of a rotating fluid in a spheroidal container
}

\author{
D. Schmitt \\ Laboratoire Louis Néel, CNRS, BP 166, 38042 Grenoble Cédex 9, France \\ and D. Jault \\ LGIT, CNRS, Université Joseph Fourier BP 53, 38041 Grenoble Cédex 9, France
}

\begin{abstract}
The motion of an incompressible, viscous rotating fluid contained in a spheroidal container is studied by a direct numerical simulation in an oblate spheroidal coordinate system. An appropriate formalism is first derived which allows us to expand any scalar field in spherical harmonics and to decompose any vector field into its sphero-poloidal and sphero-toroidal scalar parts. The spinover mode is then considered, by linearizing the equation of motion for the fluid. Boundary conditions specific of the spheroidal geometry are explicited. Finite difference method for the sphero-radial component and spherical harmonics expansion for angular components are considered. A few numerical results are given about the viscous effects on the spinover mode, for different eccentricities of the container, in good agreement with the analytical solution given in the literature.
\end{abstract}

Keywords : Rotating fluids, viscous effects, spheroidal geometry, Mie decomposition.

MSC codes : 65M99 : Partial differential equation, initial value problem.

76U05 : Rotating fluids.

\section{Corresponding author :}

SCHMITT Denys

Laboratoire Louis Néel - CNRS

BP 166, 38042 Grenoble Cedex 9, FRANCE

Tél : (33) 4.76.88.79.15

Fax : (33) 4.76.88.11.91

email : Denys.Schmitt@grenoble.cnrs.fr 


\section{1 - Introduction}

The response of a rotating viscous, incompressible fluid to the precession of its container has been subject of numerous studies since the early twentieth century, when Poincaré calculated the effects of the Earth's precession on the motion of its inner liquid core [1]. The spheroidal geometry of the rigid boundary of the latter was explicitely considered, giving rise to specific effects on the Earth's nutation compared to the case of a spherical container, but viscosity was neglected. Viscosity was later considered as a correction of the non viscous modes, with a special attention to the critical regions of the Ekman layer [2-4]. Later, following these early fundamental analytical approaches, numerical studies have been developed $[5,6]$. Indeed, such simulations are needed to go further into our knowledge of the phenomena and to explain experimental results where fluid instabilities and turbulence are observed and reveal non-linear effects [7-9]. The role of precession (and convection) for the generation of the geomagnetic field in rotating bodies has also been discussed in detail [10, $11]$.

Most of the numerical studies of rotating fluids have been developed in a spherical geometry, because of its relative simplicity. It has been the case of some geomagnetic applications [11-14]. Using a more realistic coordinate system appears however highly desirable, because natural bodies or cavities are often ellipsoidal rather than spherical. Such an approach has been used for studying kinematic dynamo models in a spheroidal galaxy [15]. More recently, a spheroidal symmetry has been considered for some geophysical applications [16-18]. In the latter approach, a coordinate transformation was performed in order to replace the ellipsoidal volume by a spherical one, for which a distorted equation of motion was used. As well, a solid inner core was included, allowing to avoid singularities at $r=0$.

In the present work, equation of motion of a rotating, viscous, incompressible fluid within a spheroidal container is directly treated in real space by using an appropriate set of coordinates, namely the oblate spheroidal coordinate system. Within this system, an extension 
of the spherical Mie representation of a vector field [19] is first considered: a solenoidal vector field (here the velocity field) is decomposed into the sum of two unique vectors which are themselves derived from two scalar fields, namely the sphero-poloidal and the spherotoroidal ones. As for the spherical symmetry, this Mie-like representation allows one to work with only two scalar fields instead of the three velocity components, once the pressure has been eliminated from the equations. In addition, an appropriate spherical harmonic expansion is used for the angular part of these spheroidal scalar fields, while a discretisation is performed for their sphero-radial component.

In a first step, precession is not included so that the problem is linear. As well, no inner core is considered. Section 2 is devoted to the mathematical developments within the oblate spheroidal coordinate system, in particular the Mie-like representation of a vector field and its properties. Then the equation of motion is rewritten as a function of both spheroidal scalar fields, and the boundary conditions are explicited (Sect. 3). In Sect. 4, as a test of our numerical procedure, the time evolution of the $(2,1,1)$ Poincaré (spinover) mode, which consists of a nearly rigid rotation about an equatorial axis, is followed for different values of eccentricity and Ekman number, and compared with analytical predictions given in the literature [4]. Perspectives are given in the last section.

\section{2 - The oblate spheroidal coordinate system}

\section{1 - Definition}

A coordinate system which appears to be particularly appropriate to the present study (spheroidal container, no inner core) is the oblate spheroidal system, related to the cartesian one by the relations: 


$$
\left\{\begin{array} { l } 
{ x = a \operatorname { c o s h } \mu \operatorname { s i n } \theta \operatorname { c o s } \varphi } \\
{ y = a \operatorname { c o s h } \mu \operatorname { s i n } \theta \operatorname { s i n } \varphi } \\
{ z = a \operatorname { s i n h } \mu \operatorname { c o s } \theta }
\end{array} \quad \text { with } \left\{\begin{array}{l}
\mu \geq 0 \\
0 \leq \theta \leq \pi \\
0 \leq \varphi<2 \pi
\end{array}\right.\right.
$$

where $a$ is the distance between the origin and the circle of foci within the equatorial plane [20]. Within this system, the constant- $\mu$ surfaces are ellipsoids with eccentricity $e=1 / \cosh \mu$, while the constant $\theta$ surfaces are one-sheet hyperboloids which, for large $\mu$ values, become asymptotically cones of revolution around the z-axis with a half-aperture $\theta$ (see Fig. 1). Some useful formulae are gathered in Appendix A.

\section{2 - Spherical harmonic expansion}

The usual expansion of a function $\mathrm{f}(\mu, \theta, \varphi)$ defined on a spheroidal surface $\mu=$ constant in spherical harmonics $Y_{l}^{m}(\theta, \varphi)$ is not directly valid here because the spheroidal colatitude $\theta$ is not the true spherical angle of $\mathrm{M}$. Considering the true spherical angle $\theta_{\mathrm{S}}$ is possible but extremely tedious because $\theta$ and $\mu$ are coupled in the expression of $\theta_{\mathrm{S}}$ (see Eq. A1). Another possibility is considering the following point transformation $\mathrm{T}_{\mathrm{P}}$ :

$$
(\mu, \theta, \varphi) \rightarrow\left(x^{\prime}, y^{\prime}, z^{\prime}\right)
$$

where the cartesian coordinates $\left(x^{\prime}, y^{\prime}, z^{\prime}\right)$ are defined as:

$$
\left\{\begin{array}{l}
x^{\prime}=\frac{a}{2} \mathrm{e}^{\mu} \sin \theta \cos \varphi \\
y^{\prime}=\frac{a}{2} \mathrm{e}^{\mu} \sin \theta \sin \varphi \\
z^{\prime}=\frac{a}{2} \mathrm{e}^{\mu} \cos \theta
\end{array}\right.
$$

The image of a spheroid $\mu=$ constant is then a sphere obtained by a contraction in the $(\mathrm{x}, \mathrm{y})$ plane together with an expansion along the $\mathrm{z}$ axis. The interest of this transformation is that the initial spheroidal $(\theta, \varphi)$ variables become the true spherical angular coordinates of the 
transformed point, its radial component being $r^{\prime}=a \mathrm{e}^{\mu} / 2$. If the new function $\mathrm{g}\left(r^{\prime}, \theta, \varphi\right) \equiv \mathrm{f}(\mu$, $\theta, \varphi)$ is defined, it is obvious that $\mathrm{g}\left(r^{\prime}, \theta, \varphi\right)$ can be now expanded in spherical harmonics in the usual manner:

$$
\mathrm{g}\left(r^{\prime}, \theta, \varphi\right)=\mathrm{f}(\mu, \theta, \varphi)=\sum_{l, m} \mathrm{~g}_{l}^{m}\left(r^{\prime}\right) \mathrm{Y}_{l}^{m}(\theta, \varphi)=\sum_{l, m} \mathrm{f}_{l}^{m}(\mu) \mathrm{Y}_{l}^{m}(\theta, \varphi)
$$

\section{3 - Decomposition of a vectorial field in the spheroidal symmetry}

In the same way as for a scalar field with regard to the spherical harmonic expansion (see above), the decomposition of a vectorial field within the Mie representation (see [19]), i.e. into a poloidal part and a toroidal part, is not appropriate in spheroidal symmetry, because it would involve the true radial $r$ variable which depends on both $\mu$ and $\theta$ in a complex manner. An alternative possibility is the following. Let us consider the vector field $\mathbf{V}(\mu, \theta, \varphi)$ $=\mathrm{V}_{\mu} \hat{\mathbf{e}}_{\mu}+\mathrm{V}_{\theta} \hat{\mathbf{e}}_{\theta}+\mathrm{V}_{\varphi} \hat{\mathbf{e}}_{\varphi}$ defined within the local spheroidal basis $\left(\hat{\mathbf{e}}_{\mu}, \hat{\mathbf{e}}_{\theta}, \hat{\mathbf{e}}_{\varphi}\right)$. Let us define the vector transformation $\mathrm{T}_{\mathbf{v}}$ :

$$
\mathbf{V}(\mu, \theta, \varphi) \rightarrow \mathbf{V}^{\prime}\left(r^{\prime}, \theta, \varphi\right)
$$

where $\left(r^{\prime}, \theta, \varphi\right)$ is defined as previously by the $\mathrm{T}_{\mathrm{P}}$ transformation (Section 2.2) and:

$$
\begin{aligned}
& \mathbf{V}^{\prime}\left(r^{\prime}, \theta, \varphi\right)=4 \cosh \mu \mathrm{e}^{-2 \mu} \sqrt{\sinh ^{2} \mu+\cos ^{2} \theta}\left(\mathrm{V}_{\mu} \hat{\mathbf{e}}_{r^{\prime}}^{\prime}+\mathrm{V}_{\theta} \hat{\mathbf{e}}_{\theta}^{\prime}\right) \\
& +4 \mathrm{e}^{-2 \mu}\left(\sinh ^{2} \mu+\cos ^{2} \theta\right) \mathrm{V}_{\varphi} \hat{\mathbf{e}}_{\varphi}^{\prime}
\end{aligned}
$$

Here $\hat{\mathbf{e}}_{r^{\prime}}^{\prime}, \hat{\mathbf{e}}_{\theta}^{\prime}$ and $\hat{\mathbf{e}}_{\varphi}^{\prime}$ are the usual spherical unit vectors at $\left(r^{\prime}, \theta, \varphi\right)$. The main property of this transformation is that the divergence of both vector fields are related to each other in a very simple way : 


$$
\nabla \cdot \mathbf{V}=\frac{\mathrm{e}^{3 \mu}}{8 \cosh \mu\left(\sinh ^{2} \mu+\cos ^{2} \theta\right)} \nabla^{\prime} \cdot \mathbf{V}^{\prime}
$$

It follows that the vanishing of $\nabla . \mathbf{V}$ implies the vanishing of $\nabla^{\prime} \cdot \mathbf{V}^{\prime}$. Therefore, assuming that the vector field $\mathbf{V}$ is solenoïdal, i.e. $\nabla \cdot \mathbf{V}=0$, the usual Mie decomposition can be applied to the vector field $\mathbf{V}^{\prime}$, and its true toroidal $\mathrm{t}^{\prime}\left(r^{\prime}, \theta, \varphi\right)$ and poloidal $\mathrm{p}^{\prime}\left(r^{\prime}, \theta, \varphi\right)$ scalar fields can then be defined as:

$$
\mathbf{V}^{\prime}\left(r^{\prime}, \theta, \varphi\right)=\nabla^{\prime} \times\left[\mathbf{r}^{\prime} \mathrm{t}^{\prime}\left(r^{\prime}, \theta, \varphi\right)\right]+\nabla^{\prime} \times\left(\nabla^{\prime} \times\left[\mathbf{r}^{\prime} \mathrm{p}^{\prime}\left(r^{\prime}, \theta, \varphi\right)\right]\right)
$$

Returning now to the initial vector field $\mathbf{V}$ by using Eq. 4 allows us to define its spherotoroidal $\mathbf{V}_{\text {st }}$ and sphero-poloidal $\mathbf{V}_{\text {sp }}$ parts, according to :

$$
\left\{\begin{array}{l}
\mathbf{V}_{\mathrm{st}}=\nabla \times\left(\frac{a}{4 \sqrt{\sinh ^{2} \mu+\cos ^{2} \theta}} \text { st } \hat{\mathbf{e}}_{\mu}\right) \\
\mathbf{V}_{\mathrm{sp}}=\nabla \times\left(\frac{1}{2 \sin \theta \sqrt{\sinh ^{2} \mu+\cos ^{2} \theta}} \hat{\mathbf{e}}_{\theta} \partial_{\varphi} \mathrm{sp}-\frac{1}{2 \cosh \mu} \hat{\mathbf{e}}_{\varphi} \partial_{\theta} \mathrm{sp}\right)
\end{array}\right.
$$

where $\partial_{\alpha}$ is a short notation for $\partial / \partial_{\alpha}$, and where $\operatorname{st}(\mu, \theta, \varphi)$ and $\operatorname{sp}(\mu, \theta, \varphi)$ are the spherotoroidal and sphero-poloidal scalar fields of $\mathbf{V}$ :

$$
\left\{\begin{array}{l}
\operatorname{st}(\mu, \theta, \varphi)=\mathrm{e}^{2 \mu} \mathrm{t}^{\prime}\left(r^{\prime}, \theta, \varphi\right) \\
\operatorname{sp}(\mu, \theta, \varphi)=\mathrm{e}^{\mu} \mathrm{p}^{\prime}\left(r^{\prime}, \theta, \varphi\right)
\end{array}\right.
$$

Taking into account the expression of the curl in the spheroidal system (see Appendix A), the three components of $\mathbf{V}$ can be written as: 


$$
\left\{\begin{array}{l}
\mathrm{V}_{\mu}=\frac{1}{2 a \cosh \mu \sqrt{\sinh ^{2} \mu+\cos ^{2} \theta}} \mathrm{L}^{2} \mathrm{sp} \\
\mathrm{V}_{\theta}=\frac{1}{4 a \cosh \mu \sin \theta \sqrt{\sinh ^{2} \mu+\cos ^{2} \theta}}\left(2 \sin \theta \partial_{\mu} \partial_{\theta} \mathrm{sp}+a \partial_{\varphi} \mathrm{st}\right) \\
\mathrm{V}_{\varphi}=\frac{1}{4 a \sin \theta\left(\sinh ^{2} \mu+\cos ^{2} \theta\right)}\left(2 \partial_{\mu} \partial_{\varphi} \mathrm{sp}-a \sin \theta \partial_{\theta} \mathrm{st}\right)
\end{array}\right.
$$

where

$$
\mathrm{L}^{2}=-\partial_{\theta}^{2}-\cot \theta \partial_{\theta}-\left(1 / \sin ^{2} \theta\right) \partial_{\varphi}^{2}
$$

is the usual angular laplacian, but written as a function of the spheroidal $\theta$ variable. A straightforward manipulation of Eq. 9 allows us to derive the spheroidal scalar fields from a given vector field:

$$
\left\{\begin{aligned}
\mathrm{L}^{2} \mathrm{sp} & =2 a \cosh \mu \sqrt{\sinh ^{2} \mu+\cos ^{2} \theta} \mathrm{V}_{\mu} \\
\mathrm{L}^{2} \mathrm{st} & =\frac{-4 \cosh \mu \sqrt{\sinh ^{2} \mu+\cos ^{2} \theta}}{\sin \theta} \partial_{\varphi} \mathrm{V}_{\theta} \\
& +\frac{4}{\sin \theta} \partial_{\theta}\left(\sin \theta\left(\sinh ^{2} \mu+\cos ^{2} \theta\right) \mathrm{V}_{\varphi}\right) \\
\partial_{\mu} \mathrm{L}^{2} \mathrm{sp} & =\frac{-2 a \cosh \mu}{\sin \theta} \partial_{\theta}\left(\sin \theta \sqrt{\sinh ^{2} \mu+\cos ^{2} \theta} \mathrm{V}_{\theta}\right) \\
& -\frac{2 a\left(\sinh \mu+\cos ^{2} \theta\right)}{\sin \theta} \partial_{\varphi} \mathrm{V}_{\varphi}
\end{aligned}\right.
$$

Note that the first two equations are sufficient to determine both scalar fields. Some similarities with the expressions for the Mie representation in spherical symmetry may then be emphasized, e.g. about the sphero-poloidal field, but it is not the general case. In particular, the curl of a sphero-poloidal field is not a sphero-toroidal field, nor the opposite assertion. 


\section{Spheroidal coordinate singularities}

In some of the above expressions, e.g. Eq. 9, a singularity occurs for particular values of spheroidal variables, namely $\theta=0$, and $(\mu=0, \theta=\pi / 2)$. This is related to the pole problem in spherical geometry, and the behaviour is worth being considered in the present study. As far as the differential operators are concerned (Appendix A), and as in spherical coordinate system, the singularities are only apparent, vanishing as soon as the expressions are written in cartesian coordinates. Other singular behaviours are discussed in Appendix B.

\section{Limit $a \rightarrow 0$}

The limit $a \rightarrow 0$ should give again the spherical situation. In that case, $\mu$ goes to infinity, and the following approximations can be made :

$$
\left\{\begin{array}{l}
a \mathrm{e}^{\mu} \approx 2 r ; a \cosh \mu \approx a \sinh \mu \approx r ; \theta \approx \theta_{\mathrm{s}} ; \partial_{\mu}=r \partial_{r} \\
\hat{\mathbf{e}}_{\mu} \approx \hat{\mathbf{e}}_{r} ; \hat{\mathbf{e}}_{\theta} \approx \hat{\mathbf{e}}_{\theta_{\mathrm{S}}} \\
\mathrm{T}_{\mathrm{P}} \approx \mathbf{1} ; \mathrm{M}^{\prime} \approx \mathrm{M} \quad ; \quad \mathrm{T}_{\mathrm{v}} \approx \mathbf{1} ; \mathbf{V}^{\prime} \approx \mathbf{V} \\
\mathrm{t}^{\prime}\left(r^{\prime}, \theta, \varphi\right) \approx \mathrm{t}\left(r, \theta_{\mathrm{s}}, \varphi\right) ; \mathrm{p}^{\prime}\left(r^{\prime}, \theta, \varphi\right) \approx \mathrm{p}\left(r, \theta_{\mathrm{s}}, \varphi\right)
\end{array}\right.
$$

and the usual expressions for spherical toroidal and poloidal fields are recovered :

$$
\begin{aligned}
\mathbf{V}_{\mathrm{st}} & =\nabla \times[\mathbf{r} \mathrm{t}(r, \theta, \varphi)] \\
\mathbf{V}_{\mathrm{sp}} & =\nabla \times(\nabla \times[\mathbf{r} \mathrm{p}(r, \theta, \varphi)])
\end{aligned}
$$

\section{3 - Mathematical formulation}

\section{1 - Equation of motion}


As a simplified stage of our work, the viscous correction to the $(2,1,1)$ (spinover) inviscid mode in spheroidal geometry is first considered, by linearizing the full equation of motion. An incompressible viscous fluid is enclosed in an oblate spheroidal container of sphero-radial coordinate $\mu_{c}$ and eccentricity $e_{c}=1 / \cosh \mu_{c}$. The container is spinning with frequency $\omega_{c}$ around the $z$ axis (no precession is considered). The linear, dimensionless equation of motion for the fluid velocity $\mathbf{u}$ in the coordinate system rotating with the container is:

$$
\frac{\partial \mathbf{u}}{\partial t}+2 \hat{\mathbf{z}} \times \mathbf{u}=-\nabla \phi+E_{\mathrm{ks}} \Delta \mathbf{u}
$$

where $\phi$ is the reduced pressure and $E_{\mathrm{ks}}=E_{\mathrm{k}} / e_{c}^{2}$ the spheroidal Ekman number. Here the focus parameter $a$ has been taken as unit of length, and $\omega_{c}^{-1}$ as unit of time.

To handle the equation of motion more easily, the pressure was first eliminated by taking the curl of Eq. 14. Projecting on $\hat{\mathbf{e}}_{\mu}$ and $\hat{\mathbf{e}}_{\varphi}$ then leads to a set of two coupled differential equations for the two remaining unknown quantities, $\operatorname{st}(\mu, \theta, \varphi)$ and $\operatorname{sp}(\mu, \theta, \varphi)$. Equation with $\hat{\mathbf{e}}_{\theta}$ is more complex and has not been used. The main complication arises from our choice of coordinate system. Indeed, terms like $\left(\sinh ^{2} \mu+\cos ^{2} \theta\right)^{n / 2}$ enter in the denominator of the starting equations. Removing them in order to use the spherical harmonic expansion requires to multiply these equations by such half-integer power quantities.

The system to be solved then involves $\mu$-derivatives up to the fourth-order, and $\theta$ - and $\varphi$-derivatives up to the fifth and fourth order, respectively. It is worth noting that the $\varphi$ derivation is straightforward when the spherical harmonic expansion is considered. Regarding the $\theta$ variable, the order of the $\theta$-derivatives can be reduced down to first order by using Eq. 10 as many times as needed, the $\mathrm{L}^{2}$ operator introduced being again well adapted to the spherical harmonic expansion. After some manipulation, the $\theta$-dependence includes numerous terms such as $\cos ^{n} \theta$ or $\cos ^{n-1} \theta \sin \theta \partial_{\theta}$, where $n$ can be as large as 7 , but no high-order $\theta$ - 
derivative. For sake of conciseness, the corresponding equations are not given here explicitely.

The next step is to use the spherical harmonic expansion for both scalar fields:

$$
\left\{\begin{array}{l}
\operatorname{st}(\mu, \theta, \varphi)=\sum_{l, m} \mathrm{st}_{l}^{m}(\mu) \mathrm{Y}_{l}^{m}(\theta, \varphi) \\
\operatorname{sp}(\mu, \theta, \varphi)=\sum_{l, m} \mathrm{sp}_{l}^{m}(\mu) \mathrm{Y}_{l}^{m}(\theta, \varphi)
\end{array}\right.
$$

Thus, the $\theta$-dependent operators, applied to a function $\mathrm{Y}_{l}^{m}$, can be expanded into the sum of $(n+1)$ spherical harmonics with the same order $m$, and degrees of same parity ranging from $l-n$ to $l+n$ :

$$
\left\{\begin{array}{l}
\cos ^{n} \theta \mathrm{Y}_{l}^{m}(\theta, \varphi)=\sum_{p=1}^{n+1} C_{n, p}(l, m) \mathrm{Y}_{l-n+2(p-1)}^{m}(\theta, \varphi) \\
\cos ^{n-1} \theta \sin \theta \partial_{\theta} \mathrm{Y}_{l}^{m}(\theta, \varphi)=\sum_{p=1}^{n+1} S_{n, p}(l, m) \mathrm{Y}_{l-n+2(p-1)}^{m}(\theta, \varphi)
\end{array}\right.
$$

All the coefficients $C_{n, p}$ and $S_{n, p}$ can be written as a function of the only first coefficient $C_{1,1}(l, m)=C(l, m)$, through recursive relations which are given in Appendix C. Their expression for some values of $n$ are also given in Appendix C. The system of equations can then be reduced to expressions which involve partial derivatives in $\mu$ and $t$ only, and are expanded in spherical harmonics. Finally, considering separately the angular part corresponding to each given $l$ value provides a set of coupled equations which include numerous contributions coming from terms with other angular parts up to $\Delta l=7$ (i.e. from $l-7$ to $l+7)$. All the expressions have been derived by using Mathematica software.

\section{2 - Boundary conditions}


A first set of equations arises from the no-slip condition $\mathbf{u}=0$ considered at the outer boundary $\mu=\mu_{c}$ of the container, i.e. for $\mu=\mu_{c}$. From the expression of the three components of $\mathbf{u}$ (Eq. 9) and using the spherical harmonics expansion for $\operatorname{st}(\mu, \theta, \varphi)$ and $\operatorname{sp}(\mu, \theta, \varphi)$ (Eq. 15), the $\mathbf{u}\left(\mu_{c}\right)=0$ condition leads to the following relations:

$$
\operatorname{st}_{l}^{m}\left(\mu_{c}\right)=0 \quad ; \quad \operatorname{sp}_{l}^{m}\left(\mu_{c}\right)=0 \quad ; \quad \partial_{\mu} \operatorname{sp}_{l}^{m}\left(\mu_{c}\right)=0
$$

A second set of equations comes from the critical disk $\mu=0$. The absence of inner core implies that $\mathbf{u}$ and its derivatives are continuous when passing through this surface, both sides of this surface corresponding to $\theta$ and $\pi-\theta$. That leads to the following parity relations for the functions $\operatorname{st}_{l}^{m}(\mu)$ and $\operatorname{sp}_{l}^{m}(\mu)$ for small values of $\mu: \operatorname{sp}_{l}^{m}(\mu)$ and $\operatorname{st}_{l}^{m}(\mu)$ must be odd if $(l+m)$ is even, and even if $(l+m)$ is odd. In the present calculation, that implies the vanishing of some quantities like $\mathrm{st}_{2 n+1}^{1}(0), \partial_{\mu} \mathrm{sp}_{2 n}^{1}(0), \partial_{\mu}^{2} \mathrm{st}_{2 n+1}^{1}(0) \ldots$

\section{3 - Numerical method}

Numerical calculation has been made by using the finite difference method for the sphero-radial variable $\mu$, with a $\tanh (n)$-grid in order to increase the number of points within the Ekman boundary layer (total number of points has been taken as $N=81$ ). For the angular variables, the spherical harmonic expansion has been truncated to $\operatorname{lmax}=64$. For the azimutal part, only $m=1$ terms are needed here (see Sect. 4$)$, since the $(m=-1)$ terms can be deduced through the general relations:

$$
\left\{\begin{array}{l}
\mathrm{st}_{l}^{-m}(\mu)=(-1)^{m}\left(\mathrm{st}_{l}^{m}(\mu)\right)^{*} \\
\operatorname{sp}_{l}^{-m}(\mu)=(-1)^{m}\left(\operatorname{sp}_{l}^{m}(\mu)\right)^{*}
\end{array}\right.
$$

The corresponding matrices consist in a penta-diagonal matrix associated with the radial grid, the elements of which are band-matrices of order lmax, arising from the coupling 
of scalar field components having different $l$ values. Moreover, due to the properties of our equations (Eq. 9 and 16), two independent groups of these components can be considered separately: the first group involves $\mathrm{sp}_{1}^{m}, \mathrm{st}_{2}^{m}, \mathrm{sp}_{3}^{m}, \mathrm{st}_{4}^{m} \ldots$, while the second one, which will be used here below, includes $\mathrm{st}_{1}^{m}, \mathrm{sp}_{2}^{m}, \mathrm{st}_{3}^{m}, \mathrm{sp}_{4}^{m} \ldots$. The implicit Crank-Nicolson scheme has been used for the diffusion term (initial value problem). For each time step, the inversion of the big penta-diagonal matrix is carried out by LU factorization and Thomas algorithm, in order to avoid keeping in memory the inverse of this matrix. The LAPACK routines have been used for manipulating the matrices.

\section{4 - Application to the $(2,1,1)$ spinover mode}

\section{1 - Description of the $(2,1,1)$ mode in spheroidal system}

The initial condition taken as the starting $(t=0)$ velocity field in the Crank-Nicolson scheme is the $(2,1,1)$ inviscid inertial (Poincaré) mode for which all the components are purely linear in the spatial coordinates $(x, y, z)$ and satisfy the no-penetration condition on the spheroidal boundary of the container $\left(\mu=\mu_{c}\right)[4,21]$ :

$$
\mathbf{u}_{211}=\frac{z}{\tanh \left(\mu_{c}\right)} \hat{\mathbf{e}}_{y}-\tanh \left(\mu_{c}\right) y \hat{\mathbf{e}}_{z}
$$

This mode corresponds to a vortical flow around the $\hat{\mathbf{e}}_{x}$ axis, with a constant-vorticity and no boundary layer. It is the eigen mode of rotation for an inviscid flow, which is excited when a fluid is in rapid rotation around the $\mathbf{z}$-axis and its rotation axis is suddenly tilted by a slight quantity [4]. Precession may be viewed as a sequence of such infinitesimal changes so that it is anticipated that this mode plays a key role in that problem. Writing Eq. 19 in the spheroidal coordinate system and expanding the corresponding scalar fields in spherical harmonics lead 
to three non-zero terms, which involve only $m= \pm 1$ (here and in the following, the index $m=$ 1 has been omitted for sake of simplicity):

$$
\left\{\begin{array}{l}
\operatorname{st}_{1}(\mu)=\frac{1}{5} a \sqrt{\frac{2 \pi}{3}}\left[-1+\cosh \left(2 \mu_{c}\right)(4+5 \cosh (2 \mu))\right] \frac{\sinh \mu}{\sinh \mu_{c} \cosh \mu_{c}} \\
\operatorname{sp}_{2}(\mu)=\frac{\mathrm{i}}{6} a^{2} \sqrt{\frac{2 \pi}{15}} \frac{\cosh (2 \mu)-\cosh \left(2 \mu_{c}\right)}{\sinh \mu_{c} \cosh \mu_{c}} \cosh \mu \\
\operatorname{st}_{3}(\mu)=\frac{16}{15} a \sqrt{\frac{\pi}{21}} \operatorname{coth} \mu_{c} \sinh \mu
\end{array}\right.
$$

It is worth noting that the boundary conditions of this inviscid Poincaré solution are verified at $\mu=0$ but not at $\mu=\mu_{c}$. The values at $\mu_{N}=\mu_{c}$ for $\mathrm{st}_{1}$ and $\mathrm{st}_{3}$, as well as the previous one at $\mu_{N-1}$ for $\mathrm{sp}_{2}$ need to be modified in order to fulfill the outer boundary conditions and to take into account the viscosity (Eq. 17). Moreover, the pure solid rotation within the spherical limit $(a=0)$ is found by applying Eq. 12 to Eq. 20 :

$$
\mathrm{st}_{1}(\mu) \approx 4 \sqrt{\frac{2 \pi}{3}} \frac{r^{3}}{a^{2}} \quad ; \quad \mathrm{sp}_{2}(\mu) \approx \frac{\mathrm{i}}{3} a r \sqrt{\frac{2 \pi}{15}} \frac{r^{2}-r_{c}^{2}}{r_{c}^{2}} \quad ; \quad \mathrm{st}_{3}(\mu) \approx \frac{16}{15} \sqrt{\frac{\pi}{21}} r
$$

or :

$$
\mathrm{t}_{1}(r) \approx \sqrt{\frac{2 \pi}{3}} r \quad ; \quad \mathrm{p}_{2}(r) \approx \frac{\mathrm{i}}{6} a^{2} \sqrt{\frac{2 \pi}{15}} \frac{r^{2}-r_{c}^{2}}{r_{c}^{2}} ; \quad \mathrm{t}_{3}(r) \approx \frac{4}{15} \sqrt{\frac{\pi}{21}} \frac{a^{2}}{r}
$$

Only the first (toroidal) term $\mathrm{t}_{1}(r)$ then remains dominant within the limit $a \rightarrow 0$, as expected for a true solid rotation.

\section{2 - Calculation of the pseudo-vorticity}

The expression of true vorticity in the spheroidal system being complex to evaluate, an alternative, more simple way to estimate the vorticity of the rotating fluid then its time dependence is to calculate its pseudo-vorticity $\omega_{\mathrm{pv}}$, which is defined as : 


$$
\omega_{p v}=\int_{\text {spheroid }} \mathbf{r} \times \mathbf{u}
$$

Within the spherical limit, this vector is nothing else than half of the true vorticity, $(1 / 2) \nabla \times \mathbf{u}$ . In spheroidal symmetry, expanding the scalar fields of any vector field $\mathbf{u}$ in spherical harmonics and integrating their angular parts leads to the existence of only two contributions, which can be written, in the cartesian basis, as:

$$
\left\{\begin{array}{l}
\omega_{p v 1}=\sqrt{\frac{\pi}{6}} \int_{0}^{\mu_{c}}\left[-\Re\left(\mathrm{st}_{1}(\mu)\right) \hat{\mathbf{e}}_{x}+\Im\left(\mathrm{st}_{1}(\mu)\right) \hat{\mathbf{e}}_{y}\right] \sinh (2 \mu) d \mu \\
\omega_{p v 2}=2 \sqrt{\frac{6 \pi}{5}} \int_{0}^{\mu_{c}}\left[\Im\left(\operatorname{sp}_{2}(\mu)\right) \hat{\mathbf{e}}_{x}+\Re\left(\operatorname{sp}_{2}(\mu)\right) \hat{\mathbf{e}}_{y}\right] d \mu
\end{array}\right.
$$

It turns out that this pseudo-vorticity $\omega_{\mathrm{pv}}$, apart a constant prefactor, is nothing else but the projection of the vector field $\mathbf{u}$ on the two orthogonal spinover modes $\mathbf{u}_{\mathbf{2 1 1}}$ and $\mathbf{u}_{\mathbf{2} \mathbf{1 1}}$ which correspond to the inertial vortical flows around $\hat{\mathbf{e}}_{x}$ and $\hat{\mathbf{e}}_{y}$, respectively:

$$
\boldsymbol{\omega}_{\mathrm{pv}}=-\operatorname{coth}\left(2 \mu_{c}\right)\left[\int_{\text {spheroid }}\left(\mathbf{u}_{\mathbf{2 1 1}}{ }^{*} \cdot \mathbf{u}\right) \hat{\mathbf{e}}_{x}+\int_{\text {spheroid }}\left(\mathbf{u}_{2 \overline{1} 1}{ }^{*} \cdot \mathbf{u}\right) \hat{\mathbf{e}}_{y}\right]
$$

The evolution of the system will then be followed by investigating the time-variation of this vector $\omega_{\mathrm{pv}}$ as well as its dependence on viscosity and eccentricity.

\section{3 - Numerical results}

The pseudo-vorticity $\omega_{\mathrm{pv}}$ can be defined by its magnitude $\omega$ and its position. As expected from a theoretical point of view, $\omega$ is found to decay exponentially with time according to viscous effects (negative decay factor $\lambda$ ), while the vector $\omega_{\mathrm{pv}}$ is found to remain within the equatorial plane, rotating in a retrograde way with negative angular speed $\omega_{d}$ : 


$$
\left\{\begin{array}{l}
\omega=\omega_{0} \exp (\lambda t) \\
\left(\omega_{\mathrm{pv}}, \hat{\mathbf{e}}_{x}\right)=\pi+\omega_{\mathrm{d}} t
\end{array}\right.
$$

where $\left(\omega_{\mathrm{pv}}, \hat{\mathbf{e}}_{x}\right)$ is the angle between vectors $\omega_{\mathrm{pv}}$ and $\hat{\mathbf{e}}_{\mathrm{x}}$. Both $\lambda$ and $\omega_{d}$ have been followed over several decades of the Ekman number $E_{\mathrm{k}}$, as shown in Fig. 2 for two different eccentricities, namely $e=0.02$ and 0.266 . The former value has been chosen close to zero to compare with the spherical situation, while the other one is noticeably different from zero to appreciate the effects of ellipticity. In both cases, the expansion of $\lambda$ in power of $E_{k}^{1 / 2}$ is approached for low Ekman numbers, while $\omega_{d}$ tends to saturate toward a constant which differs from unity as soon as the eccentricity is different from zero. The next term in the $E_{k}^{1 / 2}$ expansion of $\lambda$ and $\omega_{d}$ is hard to appreciate, because of the limited accuracy of these preliminary results. Moreover, the same limitation applies for explaining the dispersion of the calculated values at low Ekman numbers where a much higher radial resolution would be needed. It is worth noting that the inner core, which is often included in spherical symmetry in order to avoid a singularity at the origin, is not considered here. In the spheroidal geometry, the singularity is reported to the focus circle, but the effects of this singular circle seem to be rather limited in the present linear approach where only $m=1$ terms are included.

Calculations have been performed for several values of eccentricity ranging from 0.02 to 0.5 . Initial $e^{2}$ dependence is found (see Fig. 3), as expected from previous theoretical analysis $[2,4,22]$ :

$$
\left\{\begin{array}{l}
\lambda=\lambda_{l}(e) E_{k}{ }^{1 / 2}+O\left(E_{k}, e^{4} E_{k}{ }^{1 / 2}\right) \\
\omega_{d}=\omega_{0}(e)+\omega_{l}(e) E_{k}{ }^{1 / 2}+\ldots \\
\text { with } \lambda_{l}(e)=-2.62-0.426 e^{2}, \\
\omega_{0}(e)=\frac{-2}{2-e^{2}} \text { and } \omega_{1}(e)=-0.258-0.766 e^{2}
\end{array}\right.
$$


The presence of viscosity implies the growing of sphero-toroidal and sphero-poloidal scalars of degree higher than the initial values 3 and 2, respectively, through the coupling of different $l$ values. All these harmonics allow us to account for the existence of internal shear layers spawn by the Ekman layer close to the outer boundary, the thickness of which scales as $E_{k}^{1 / 2}$ for all degrees, as shown in Fig. 4 for some low-order functions. At last, radial and angular resolution has been checked. The angular convergence seems to be exponential, as no significative change occurs for $\lambda$ and $\omega_{d}$ between $\operatorname{lmax}=32$ and 80 . For the radial resolution, calculations performed for $N$ ranging from 81 to 231 are consistent with a quadratic convergence.

\section{5 - Perspectives}

The present preliminary results of a numerical study of the spinover mode in spheroidal geometry validate the choice of the method which is developed here. The complexity inherent to the spheroidal symmetry (e.g. the non-separation of $\mu$ and $\theta$ variables in most of the spheroidal expressions) compared to the relative simplicity of the spherical case has been overcome by using a spheroidal decomposition of the velocity field derived from the spherical Mie representation. It is again emphasized that the present numerical results are only an illustration of the formalism developed here to work in a spheroidal geometry. The present work needs then to be continued by increasing the spatial resolution, in order to accurately describe the internal shear layers and their eccentricity dependences. Indeed, the $e=0$ case appears quite singular as far as the behaviour of these internal layers is concerned, and it is worth investigating how they will be modified by a spheroidal boundary. Another challenge will be to include precession (non-linear) effects within the present formalism, in order to be able to explain some features observed experimentally, such as fluid instabilities, axial shear structures... $[8,9,23]$. For such a non-linear study, $m \neq 1$ terms will have to be considered in the motion equation and the pole problem carefully examined. The true vorticity will have to be evaluated, giving rise to expressions more complex than in the present study. At last, realistic geophysical applications would require to include an inner boundary with a possibly 
different eccentricity, which would demand to extend the present coordinate system but would simplify the inner boundary condition.

\section{Acknowledgments :}

P. Cardin, H.C. Nataf and the Geodynamo team of the LGIT are gratefully acknowledged for their constant help during this study. Most of the computations presented in this paper were performed at the Service Commun de Calcul Intensif de l'Observatoire de Grenoble (SCCI). 


\section{APPENDIX A}

In this appendix, some useful relations are given for the spheroidal coordinate system. The connection with the spherical coordinate system $\left(r, \theta_{\mathrm{s}}, \varphi_{\mathrm{s}}\right)$ is the following:

$$
\left\{\begin{array}{l}
r=a \sqrt{\sinh ^{2} \mu+\sin ^{2} \theta} \\
\tan \theta_{\mathrm{s}}=\frac{\tan \theta}{\tanh \mu} \\
\varphi_{\mathrm{s}}=\varphi
\end{array}\right.
$$

Within the oblate spheroidal system, metric coefficients involved in the volume element $\mathrm{dV}$ can be written as:

$\left\{\begin{array}{l}h_{\mu}=h_{\theta}=a \sqrt{\sinh ^{2} \mu+\cos ^{2} \theta} \\ h_{\varphi}=a \cosh \mu \sin \theta\end{array}\right.$

whence:

$\mathrm{dV}=h_{\mu} h_{\theta} h_{\varphi} \mathrm{d} \mu \mathrm{d} \theta \mathrm{d} \varphi=a^{3} \cosh \mu \sin \theta\left(\sinh ^{2} \mu+\cos ^{2} \theta\right) \mathrm{d} \mu \mathrm{d} \theta \mathrm{d} \varphi$

Spheroidal unit vectors can be defined as a function of cartesian ones as:

$$
\left\{\begin{array}{l}
\hat{\mathbf{e}}_{\mu}=\frac{\sinh \mu \sin \theta \cos \varphi}{\sqrt{\sinh ^{2} \mu+\cos ^{2} \theta}} \hat{\mathbf{e}}_{x}+\frac{\sinh \mu \sin \theta \sin \varphi}{\sqrt{\sinh ^{2} \mu+\cos ^{2} \theta}} \hat{\mathbf{e}}_{y}+\frac{\cosh \mu \cos \theta}{\sqrt{\sinh ^{2} \mu+\cos ^{2} \theta}} \hat{\mathbf{e}}_{z} \\
\hat{\mathbf{e}}_{\theta}=\frac{\cosh \mu \cos \theta \cos \varphi}{\sqrt{\sinh ^{2} \mu+\cos ^{2} \theta}} \hat{\mathbf{e}}_{x}+\frac{\cosh \mu \cos \theta \sin \varphi}{\sqrt{\sinh ^{2} \mu+\cos ^{2} \theta}} \hat{\mathbf{e}}_{y}-\frac{\sinh \mu \sin \theta}{\sqrt{\sinh ^{2} \mu+\cos ^{2} \theta}} \hat{\mathbf{e}}_{z} \\
\hat{\mathbf{e}}_{\varphi}=-\sin \varphi \hat{\mathbf{e}}_{x}+\cos \varphi \hat{\mathbf{e}}_{y}
\end{array}\right.
$$

Finally, differential operators of interest are gradient, divergence, laplacian and curl:

$$
\nabla=\frac{1}{a \sqrt{\sinh ^{2} \mu+\cos ^{2} \theta}} \hat{\mathbf{e}}_{\mu} \partial_{\mu}+\frac{1}{a \sqrt{\sinh ^{2} \mu+\cos ^{2} \theta}} \hat{\mathbf{e}}_{\theta} \partial_{\theta}+\frac{1}{a \cosh \mu \sin \theta} \hat{\mathbf{e}}_{\varphi} \partial_{\varphi}
$$




$$
\begin{aligned}
& \nabla \cdot \mathbf{V}=\frac{1}{a \cosh \mu\left(\sinh ^{2} \mu+\cos ^{2} \theta\right)} \partial_{\mu}\left(\cosh \mu \sqrt{\sinh ^{2} \mu+\cos ^{2} \theta} \mathrm{V}_{\mu}\right) \\
& +\frac{1}{a \sin \theta\left(\sinh ^{2} \mu+\cos ^{2} \theta\right)} \partial_{\theta}\left(\sin \theta \sqrt{\sinh ^{2} \mu+\cos ^{2} \theta} \mathrm{V}_{\theta}\right)+\frac{1}{a \cosh \mu \sin \theta} \partial_{\varphi}\left(\mathrm{V}_{\varphi}\right) \\
& \Delta=\frac{1}{a^{2}\left(\sinh ^{2} \mu+\cos ^{2} \theta\right)}\left[\frac{1}{\cosh \mu} \partial_{\mu}\left(\cosh \mu \partial_{\mu}\right)+\frac{1}{\sin \theta} \partial_{\theta}\left(\sin \theta \partial_{\theta}\right)\right] \\
& \quad+\frac{1}{a^{2} \cosh ^{2} \mu \sin ^{2} \theta} \partial_{\varphi}{ }^{2} \\
& =\frac{1}{a^{2}\left(\sinh ^{2} \mu+\cos ^{2} \theta\right)}\left[\frac{1}{\cosh \mu} \partial_{\mu}\left(\cosh \mu \partial_{\mu}\right)-\frac{1}{\cosh ^{2} \mu} \partial_{\varphi}{ }^{2}-\mathbf{L}^{2}\right]
\end{aligned}
$$

where $\mathrm{L}^{2}$ is the usual angular laplacian:

$$
\mathrm{L}^{2}=-\frac{1}{\sin \theta} \partial_{\theta}\left(\sin \theta \partial_{\theta}\right)-\frac{1}{\sin ^{2} \theta} \partial_{\varphi}^{2}
$$

$\nabla \times \mathbf{V}=$

$\frac{1}{a \cosh \mu \sin \theta \sqrt{\sinh ^{2} \mu+\cos ^{2} \theta}} \hat{\mathbf{e}}_{\mu}\left[\partial_{\theta}\left(\cosh \mu \sin \theta \mathrm{V}_{\varphi}\right)-\partial_{\varphi}\left(\sqrt{\sinh ^{2} \mu+\cos ^{2} \theta} \mathrm{V}_{\theta}\right)\right]$

$-\frac{1}{a \cosh \mu \sin \theta \sqrt{\sinh ^{2} \mu+\cos ^{2} \theta}} \hat{\mathbf{e}}_{\theta}\left[\partial_{\mu}\left(\cosh \mu \sin \theta \mathrm{V}_{\varphi}\right)-\partial_{\varphi}\left(\sqrt{\sinh ^{2} \mu+\cos ^{2} \theta} \mathrm{V}_{\mu}\right)\right]$

$+\frac{1}{a\left(\sinh ^{2} \mu+\cos ^{2} \theta\right)} \hat{\mathbf{e}}_{\varphi}\left[\partial_{\mu}\left(\sqrt{\sinh ^{2} \mu+\cos ^{2} \theta} \mathrm{V}_{\theta}\right)-\partial_{\theta}\left(\sqrt{\sinh ^{2} \mu+\cos ^{2} \theta} \mathrm{V}_{\mu}\right)\right]$

In all these expressions, $\partial_{\alpha}$ stands for $\partial / \partial_{\alpha}(\alpha=\mu, \theta$ or $\varphi)$.

For sake of completeness, we report here below the spheroidal rate-of-strain components: 


$$
\left\{\begin{array}{l}
\varepsilon_{\mu \theta}=\frac{1}{a} \partial_{\mu}\left(\frac{\mathrm{V}_{\theta}}{\sqrt{\sinh ^{2} \mu+\cos ^{2} \theta}}\right)+\frac{1}{a} \partial_{\theta}\left(\frac{\mathrm{V}_{\mu}}{\sqrt{\sinh ^{2} \mu+\cos ^{2} \theta}}\right) \\
\varepsilon_{\mu \varphi}=\frac{\cosh \mu}{a \sqrt{\sinh ^{2} \mu+\cos ^{2} \theta}} \partial_{\mu}\left(\frac{\mathrm{V}_{\varphi}}{\cosh \mu}\right)+\frac{1}{a \cosh \mu \sin \theta} \partial_{\varphi}\left(\mathrm{V}_{\mu}\right) \\
\varepsilon_{\theta \varphi}=\frac{\sin \theta}{a \sqrt{\sinh ^{2} \mu+\cos ^{2} \theta}} \partial_{\theta}\left(\frac{\mathrm{V}_{\varphi}}{\sin \theta}\right)+\frac{1}{a \cosh \mu \sin \theta} \partial_{\varphi}\left(\mathrm{V}_{\theta}\right)
\end{array}\right.
$$




\section{APPENDIX B}

\section{Spheroidal coordinate singularities}

A first critical behaviour could be related to the densification of a grid near the poles if a grid was used in real space. However, as in spherical geometry, this problem could be fixed by considering the spherical harmonic expansion (see Section 2.2), at least for values of $\mu$ not too close to zero. From Eq. 2, it turns out that the length element ds along a constant- $\mu$ line as a function of $\theta$ is related to the corresponding element ds' after transformation $\mathrm{T}_{\mathrm{P}}$ by:

$$
\mathrm{ds}^{\prime}=\frac{r^{\prime}}{a \sqrt{\sinh ^{2} \mu+\cos ^{2} \theta}} \mathrm{ds}=\frac{e^{\mu}}{2 \sqrt{\sinh ^{2} \mu+\cos ^{2} \theta}} \mathrm{ds}
$$

Therefore, ds'/ds remains within the interval $[1-\exp (-\mu) / 2 \cosh (\mu), 1+\exp (-\mu) / 2 \sinh (\mu)]$, which diverges only near $\mu=0$. If a uniform grid is considered when the spherical harmonic expansion is performed, i.e. $\mathrm{ds}^{\prime}=$ constant, the grid ds in real space will remain moderately distorted, except near the critical focal circle $(\mu=0, \theta=\pi / 2)$. This situation will have to be carefully examined if a grid is used (this is not the present case).

Another critical behaviour occurs for the expression of vectors around $\theta=0$ (Eq. 9), as the unit vectors $\hat{\mathbf{e}}_{\mu}$ and $\hat{\mathbf{e}}_{\theta}$ are no longer defined. However, returning back to the cartesian coordinate system and performing an expansion of $\mathrm{V}_{\mathrm{x}}, \mathrm{V}_{\mathrm{y}}, \mathrm{V}_{\mathrm{z}}$ as a function of $\theta$ allows one to raise the singularities. After expanding the sp and st scalars in spherical harmonics, the following expressions are obtained for the limit of a vector $\mathbf{V}$ on the $\mathbf{z}$-axis in the present case $(\mathrm{m}=1)$ : 


$$
\left\{\begin{array}{l}
\mathrm{V}_{x}=\frac{-1}{2 a \cosh ^{2} \mu} \sum_{l} \partial_{\theta} Y_{l}^{1}(0,0)\left[a \mathfrak{s} \mathrm{st}_{l}(\mu)-2 \Re \partial_{\mu} \mathrm{sp}_{l}(\mu)\right] \\
\mathrm{V}_{y}=\frac{-1}{2 a \cosh ^{2} \mu} \sum_{l} \partial_{\theta} Y_{l}^{1}(0,0)\left[a \Re \mathrm{st}_{l}(\mu)+2 \mathfrak{s} \partial_{\mu} \operatorname{sp}_{l}(\mu)\right] \\
\mathrm{V}_{z}=0
\end{array}\right.
$$

At last, a critical behaviour occurs for the expression of vectors around the focal circle $(\mu=0, \theta=\pi / 2)$, where the unit vectors $\hat{\mathbf{e}}_{\mu}$ and $\hat{\mathbf{e}}_{\theta}$ are no longer defined. However, the same procedure as above allows one to raise the singularities and to obtain the following expressions for the limit of a vector $\mathbf{V}$ :

$$
\left\{\begin{array}{l}
\mathrm{V}_{x}=\mathrm{V}_{y}=0 \\
\mathrm{~V}_{z}=-\sum_{l} l(l+1) \Re\left[\operatorname{sp}_{l}(\mu) \partial_{\theta} Y_{l}^{1}\left(\frac{\pi}{2}, \varphi\right)\right]
\end{array}\right.
$$




\section{APPENDIX C}

In Eq. 16, all the coefficients $C_{n, p}(l, m)$ and $S_{n, p}(l, m)$ can be expressed as a function of the only coefficient $C_{1,1}(l, m)=C(l, m)$, according to the relations given here below. It is worth noting that these relations can be obtained step by step by using the following equations:

$\cos ^{n} \theta Y_{l}^{m}(\theta, \varphi)=\cos \theta\left(\cos ^{n-1} \theta Y_{l}^{m}(\theta, \varphi)\right)$

$\cos ^{n-1} \theta \sin \theta \quad \partial_{\theta} Y_{l}^{m}(\theta, \varphi)=\cos \theta\left(\cos ^{n-2} \theta \sin \theta \quad \partial_{\theta} Y_{l}^{m}(\theta, \varphi)\right)$

A straightforward manipulation allows then to deduce the following recursive relations:

$C_{n, 1}(l, m)=C(l-n+1, m) C_{n-1,1}(l, m)$

$C_{n, n+1}(l, m)=C(l+n, m) C_{n-1, n}(l, m)$

and for $1<p<n+1$ :

$C_{n, p}(l, m)=C(l-n+2 p-1, m) C_{n-1, p}(l, m)+C(l-n+2 p-2, m) C_{n-1, p-1}(l, m)$

The same expressions are valid if the $C_{n, p}(l, m)$ 's are replaced by $S_{n, p}(l, m)$. That leads to the following relations for values up to $n=3$ :

$$
\begin{aligned}
& C_{1,1}(l, m)=C(l, m)=\sqrt{\frac{(l+m)(l-m)}{(2 l+1)(2 l-1)}} \\
& C_{1,2}(l, m)=C(l+1, m) \\
& C_{2,1}(l, m)=C(l, m) C(l-1, m) \\
& C_{2,2}(l, m)=C(l, m)^{2}+C(l+1, m)^{2} \\
& C_{2,3}(l, m)=C(l+1, m) C(l+2, m) \\
& C_{3,1}(l, m)=C(l, m) C(l-1, m) C(l-2, m) \\
& C_{3,2}(l, m)=C(l, m) \sum_{k=l-1}^{l+1} C(k, m)^{2} \\
& C_{3,3}(l, m)=C(l+1, m) \sum_{k=l}^{l+2} C(k, m)^{2} \\
& C_{3,4}(l, m)=C(l+1, m) C(l+2, m) C(l+3, m)
\end{aligned}
$$




$$
\begin{aligned}
& S_{1,}(l, m)=-(l+1) C(l, m) \\
& S_{12}(l, m)=l C(l+1, m) \\
& S_{2,}(l, m)=-(l+1) C(l, m) C(l-1, m) \\
& S_{2,2}(l, m)=-(l+1) C(l, m)^{2}+l C(l+1, m)^{2} \\
& S_{2,3}(l, m)=l C(l+1, m) C(l+2, m) \\
& S_{3,}(l, m)=-(l+1) C(l, m) C(l-1, m) C(l-2, m) \\
& S_{3,2}(l, m)=C(l, m)\left(l \quad C(l+1, m)^{2}-(l+1) \sum_{k=l-1}^{l} C(k, m)^{2}\right) \\
& S_{3,3}(l, m)=C(l+1, m)\left(-(l+1) C(l, m)^{2}+l \sum_{k=l+1}^{l+2} C(k, m)^{2}\right) \\
& S_{3,4}(l, m)=l C(l+1, m) C(l+2, m) C(l+3, m)
\end{aligned}
$$




\section{FIGURE CAPTIONS}

Fig. 1: Sketch of the spheroidal coordinate system $(\mu, \theta, \varphi)$ in a meridian plane: $\mathrm{F}$ is the focus; solid line: constant $\mu$ curve; dotted line: constant $\theta$ curve; dashed line: asymptote of the constant $\theta$ curve for large $\mu$ values; note that the spherical colatitude of the asymptote is equal to the spheroidal colatitude of $\mathrm{M}$.

Fig. 2: Calculated variation of the decay factor $\lambda$ (left scale) and eigenfrequency $\omega_{\mathrm{d}}$ (right scale) of the spinover mode for Ekman numbers $E_{k}$ ranging from $10^{-9}$ to $10^{-4}$ and eccentricities $e=0.02$ (left hand side) and 0.266 (right hand side); lines are least square fits; horizontal dotted lines are the theoretical limits for $E_{k}=0$.

Fig. 3: Eccentricity dependence of the decay factor $\lambda_{1}$ (left scale) and eigenfrequency $\omega_{0}$ (right scale) for the spinover mode (first terms in the $E_{k}^{1 / 2}$ expansion); lines are theoretical predictions.

Fig. 4: Radial structure of the viscous boundary layer, seen through the scaling of the first four sphero-toroidal radial components for $e=0.266$; successive curves correspond to $E_{k}=7.10^{-5}$ to 7 . $10^{-9}$ (factor 10 between two successive values); the scaled radial coordinate is $\left(\mu_{\mathrm{c}}\right.$ $\mu) .\left(E_{k}\right)^{-1 / 2}\left(\mu_{\mathrm{c}}=2\right.$ for $\left.e=0.266\right)$. 


\section{REFERENCES}

[1] R. Poincaré, Sur la précession des corps déformables, Bull. Astr. 27, 321 (1910)

[2] K. Stewartson and P. H. Roberts, On the motion of a liquid in a spheroidal cavity of a precessing rigid body, J. Fluid Mech. 17, 1 (1963)

[3] F. H. Busse, Steady fluid flow in a precessing spheroidal shell, J. Fluid Mech. 33, 739 (1968)

[4] H. P. Greenspan, in: The theory of rotating fluids, (Cambridge University Press, Cambridge, 1968)

[5] R. Hollerbach and R. R. Kerswell, Oscillatory internal shear layers in rotationg and precessing flows, J. Fluid Mech. 298, 327 (1995)

[6] L. Quartapelle and M. Verri, On the spectral solutions of the 3-dimensional NavierStokes equations in spherical and cylindrical regions, Computer Physics Communications 90, $1(1995)$

[7] W. V. R. Malkus, Precession of the Earth as the cause of geomagnetism, Science 160, $259(1968)$

[8] J. P. Vanyo, P. Wilde, P. Cardin and P. Olson, Experiments on precessing flows in the Earth's liquid core, Geophys. J. Int. 121, 136 (1995)

[9] J. Noir, P. Cardin, D. Jault and J. P. Masson, Experimental evidence of nonlinear resonance effects between retrograde precession and the tilt-over mode within a spheroid, Geophys. J. Int. 154, 407 (2003)

[10] W. V. R. Malkus, Energy sources for planetary dynamos, in: Lectures on solar and planetary dynamos, edited by M. R. E. Proctor and A. D. Gilbert, (Cambridge University Press, Cambridge, 1994), p. 161. 
[11] A. Tilgner, Magnetohydrodynamic flow in precessing spherical shells, J. Fluid Mech. 379, 303 (1999)

[12] U. R. Christensen, J. Aubert, P. Cardin, E. Dormy, S. Gibbons, G. A. Glatzmaier, E.

Grote, Y. Honkura, C. Jones, M. Kono, M. Matsushima, A. Sakuraba, F. Takahashi, A.

Tilgner, J. Wicht and K. Zhang, A numerical dynamo benchmark, Phys. Earth Planet. Inter. 128, $25(2001)$

[13] A. Tilgner and F. H. Busse, Fluid flows in precessing spherical shells, J. Fluid Mech. 426, 387 (2001)

[14] J. Noir, D. Jault and P. Cardin, Numerical study of the motions within a slowly precessing sphere at low Ekman number, J. Fluid Mech. 437, 283 (2001)

[15] M. R. Walker and C. F. Barenghi, High resolution numerical dynamos in the limit of a thin disk galaxy, Geophys. Astrophys. Fluid Dyn. 76, 265 (1994)

[16] A. Tilgner, Non-axisymmetric shear layers in precessing fluid ellipsoidal shells, Geophys. J. Int. 136, 629 (1999)

[17] S. Lorenzani and A. Tilgner, Fluid instabilities in precessing spheroidal cavities, $J$. Fluid Mech. 447, 111 (2001)

[18] S. Lorenzani and A. Tilgner, Inertial instabilities of fluid flow in precessing spheroidal shells, J. Fluid Mech. 492, 363 (2003)

[19] G. Backus, R. Parker and C. Constable, Foundations of Geomagnetism, (Cambridge University Press, Cambridge, 1996)

[20] P. M. Morse and H. Feshbach, Methods of theoretical physics, (McGraw-Hill Book Company, Inc., New-York, 1953)

[21] R. R. Kerswell, The instability of precessing flow, Geophysical Astrophysical Fluid Dynamics 72, 107 (1993) 
[22] P. H. Roberts and K. Stewartson, On the motion of a liquid in a spheroidal cavity of a precessing rigid body : II, Proc. Cambridge Phil. Soc. 61, 279 (1965)

[23] J. Noir, D. Brito, K. Aldridge and P. Cardin, Experimental evidence of inertial waves in a precessing spheroidal cavity, Geophys. Res. Lett. 28, 3785 (2001) 


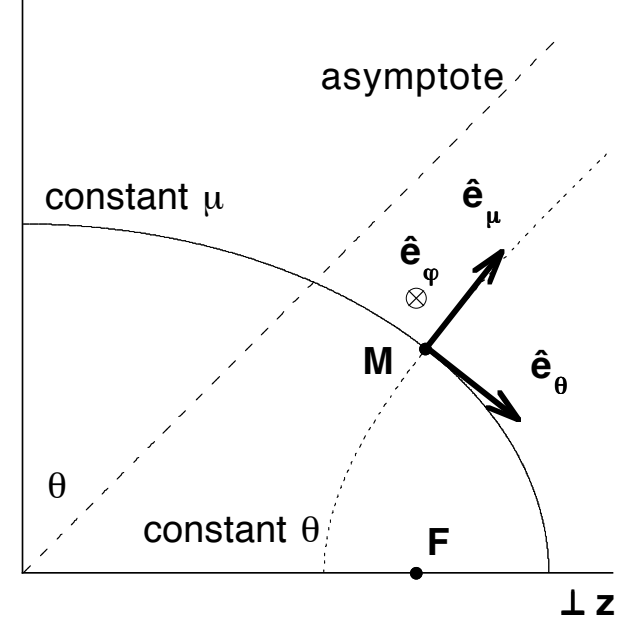



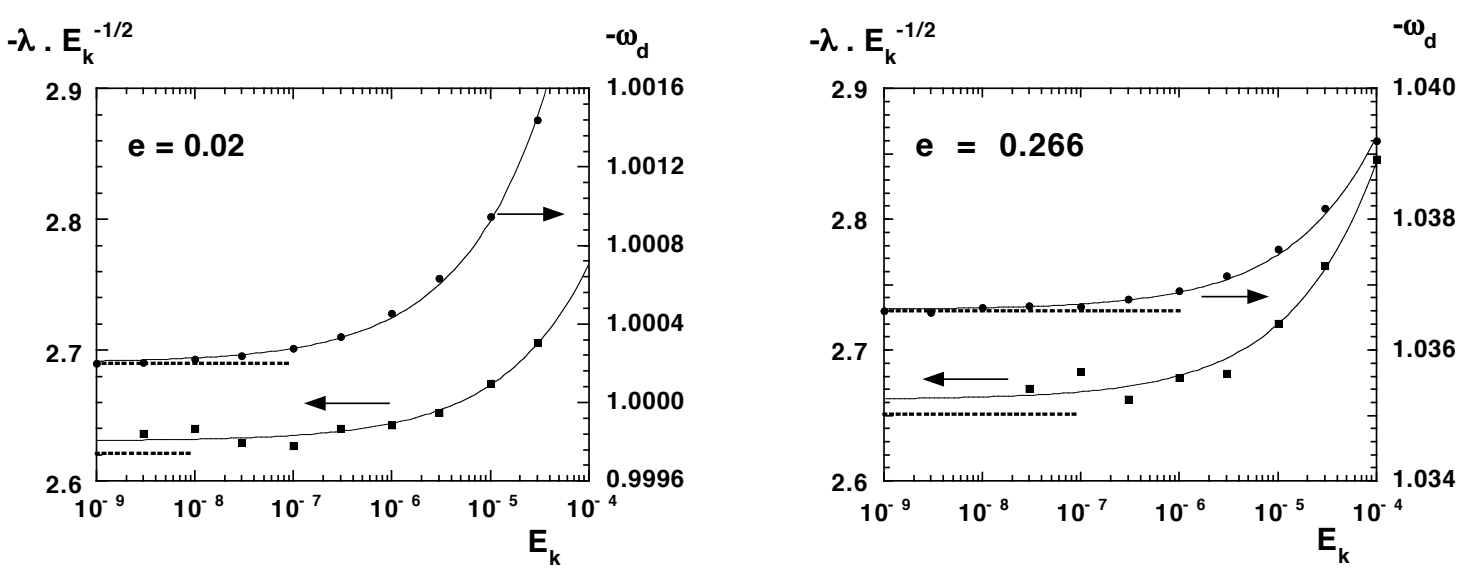


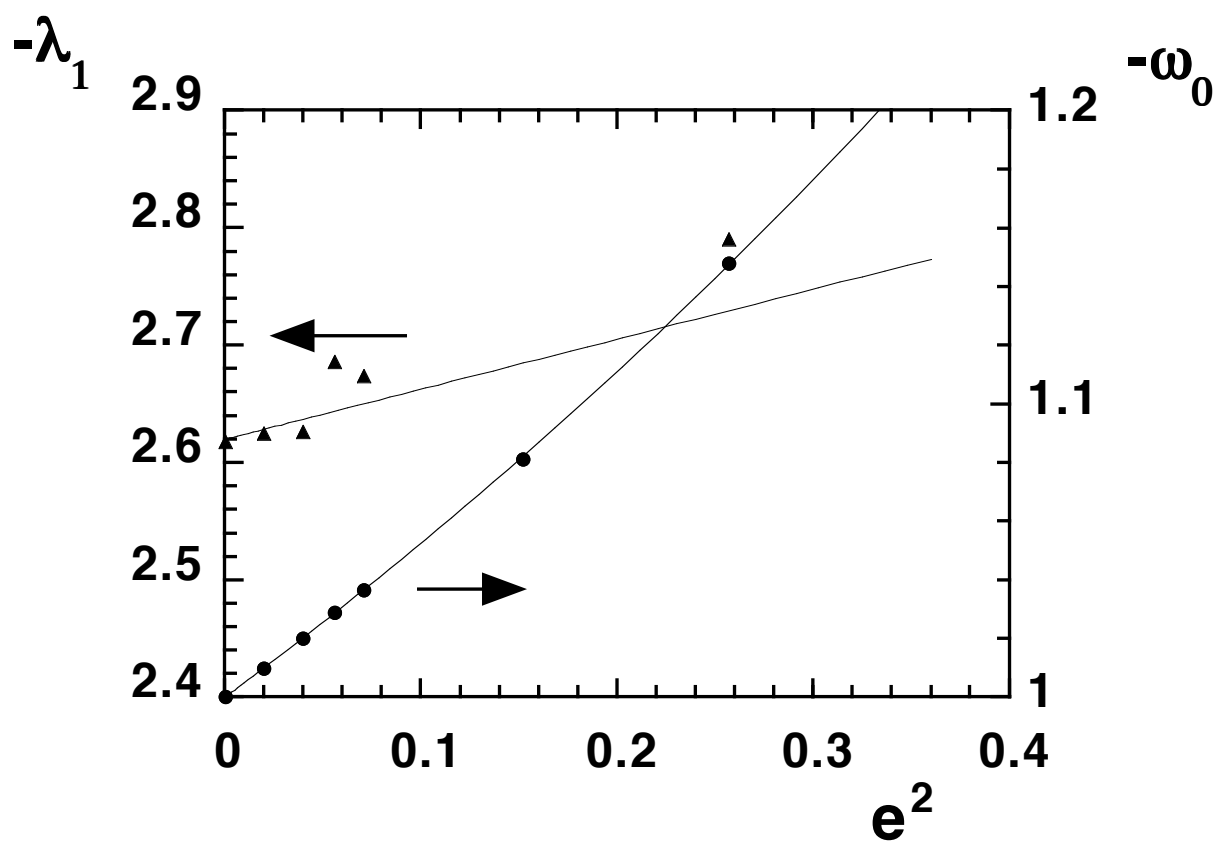



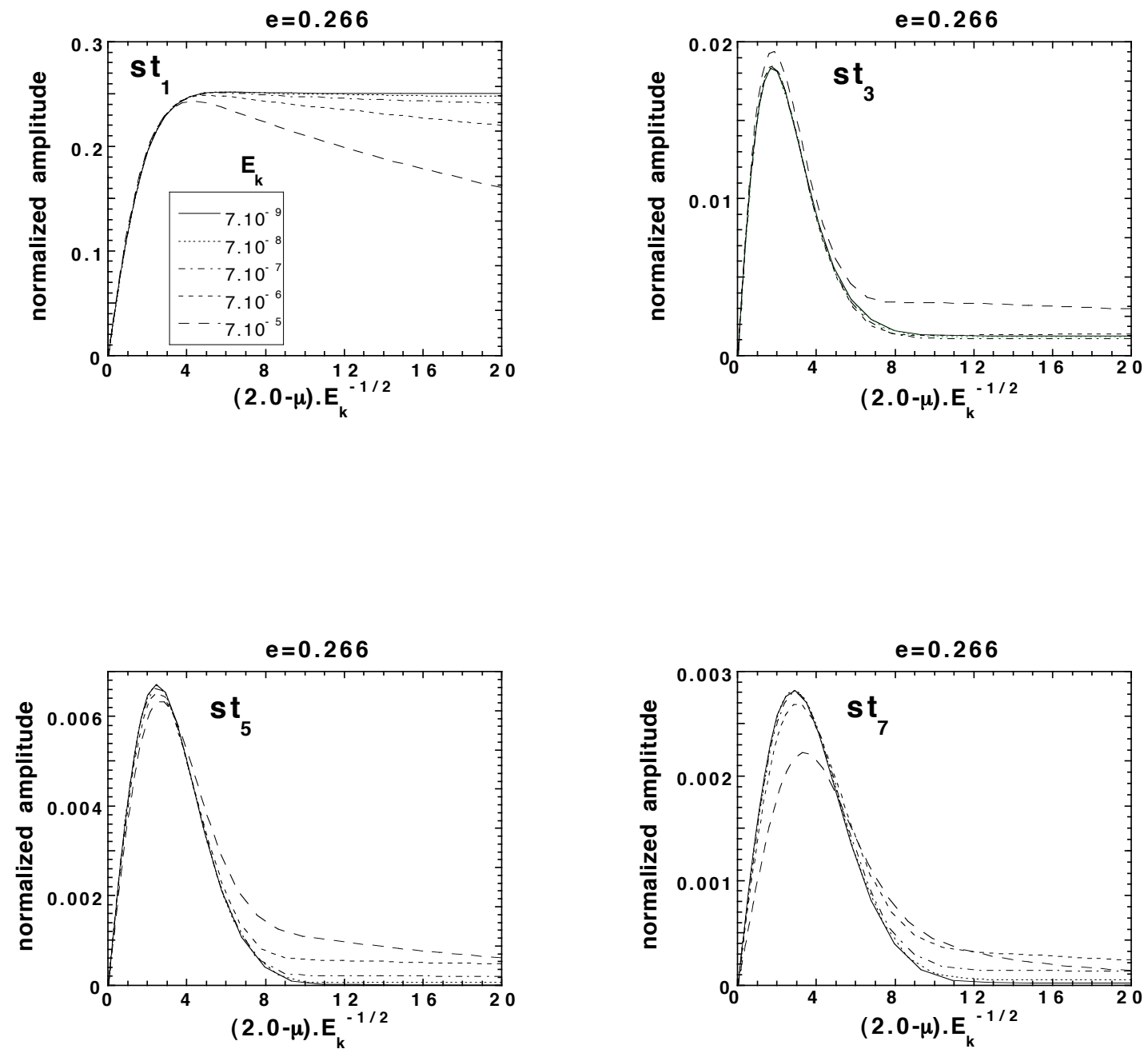